\title{
A SELECTIVE HARQ SCHEME OPERATING BASED ON CHANNEL CONDITIONS FOR HIGH SPEED PACKET DATA TRANSMISSION SYSTEMS
}

\author{
Minjae Park ${ }^{1}$, Byungjik Keum ${ }^{2}$, Moohong Lee ${ }^{2}$ and Hwang Soo Lee ${ }^{1}$ \\ ${ }^{1}$ Department of EECS, Division of Electrical Engineering, KAIST \\ 373-1, Guseong-dong, Yuseong-gu, Daejeon, 305-701, Republic of Korea \\ Tel: +82-42-869-5428, Fax: +82-42-869-8670 \\ ${ }^{2}$ Mobile Media Platform Center, KAIST \\ 373-1, Guseong-dong, Yuseong-gu, Daejeon, 305-701, Republic of Korea \\ Tel : +82-42-869-8881, Fax : +82-42-869-8880 \\ E-mail: mjpark@mcl.kaist.ac.kr, \{coolcoolbj, wildgoosemh\}@mmpc.kaist.ac.kr, hwanglee@ee.kaist.ac.kr
}

\begin{abstract}
A selective hybrid automatic repeat request (HARQ) scheme operating based on channel conditions for high speed packet data transmission systems is presented. This scheme exploits the channel condition identified by the channel quality indicator (CQI) to determine whether the retransmitted packet is directly decoded without soft combining or it is soft combined with the original packet and decoded. Since the channel condition is already measured and mapped to the CQI number by the receiver to report to the base-station for adaptive modulation and coding, additional increase of computational complexity at the receiver is not required for this scheme. The proposed selective HARQ scheme shows about two to three times performance improvement in terms of throughput because it takes the quality of retransmitted packets into account based on channel conditions before performing soft combining with the original packet with error.
\end{abstract}

\section{INTRODUCTION}

Wireless internet service with broad bandwidth and high mobility capabilities is in great demand. Therefore, high speed packet data transmission systems such as the high speed downlink packet access (HSDPA) system specified in [1], [2] are being deployed in several countries. The HSDPA system uses the adaptive modulation and coding (AMC), fast scheduling, and the hybrid automatic repeat request (HARQ) to increase data throughput of the system. Other characteristics of the HSDPA system are the small spreading factor of 16, multi-level modulation such as quadrature phase shift keying and 16 quadrature amplitude modulation, and multi-code operation in which more channelization codes are assigned according to channel conditions. When the data signal with these characteristics goes through multi-path fading channels, the multiple access interference (MAI) is generated because multi-path fading channels destroy the orthogonality of channelization codes. In turn, the MAI deteriorates the bit error rate (BER) performance of the receivers. On the other hand, the AMC in the HSDPA system operates based on channel conditions identified by the channel quality indicator (CQI) which is measured and reported by the receiver to the base-station (BS). However, time delay and the error occurring in the process of CQI reporting increase error in the receiving process. In order to complement this property of the AMC and help forward error correction to compensate for errors caused by the MAI, the HARQ scheme is used in the HSDPA system.

Several soft combining methods are used in conjunction with the HARQ scheme. In the normal HARQ scheme, when the receiver receives a retransmitted packet, it combines the retransmitted one with the original packet with error regardless of the quality of the retransmitted packet. As a result, in case the retransmitted packet has no error, the process of soft combining the retransmitted packet with the original packet with error results in poor receiver's performance compared to the case that only the retransmitted packet without error is used for the remaining signal processing without soft combining with the original packet.

In this paper, we propose a selective HARQ scheme exploiting channel conditions identified by the CQI number to determine whether the retransmitted packet is directly decoded without soft combining or it is soft combined with the original packet with error and decoded. Since the proposed scheme takes the quality of retransmitted packets into account based on channel conditions before performing soft combining with the original packet with error, it shows about two to three times performance improvement in terms of throughput. In addition, since the CQI number is already measured and reported to the BS by the receiver for the AMC, additional increase of computational complexity at the receiver is not necessary for this scheme.

\section{SOFT COMBINING METHODS FOR THE HARQ SCHEME}

The HARQ scheme complements a link adaptation process by masking inaccuracies reported in the measured channel quality of user equipments. The HARQ process comprises several packet transmissions and retransmissions. If a decoded packet passes the CRC check, the receiver returns an acknowledgement (ACK) message and the transmitter that receives the ACK message transmits another new packet. And if the decoded packet fails the CRC check, the receiver returns a negative acknowledgement (NACK) message and the transmitter which receives the NACK message retransmits the packet according to the specified combining method. Normally, chase combining method and incremental redundancy (IR) method are used for the HARQ combining in the high speed packet data transmission such as the HSDPA system specified in [1], [2], [5]. 


\section{A. Chase combining}

In chase combining method as shown in Fig. 1, when a transmitter receives a NACK message, it retransmits the packet with the same data and parity bit pattern. If the receiver receives a retransmitted packet, it combines the original transmitted packet with CRC error with the retransmitted one. The chase combining method improves performance due to better SNR.
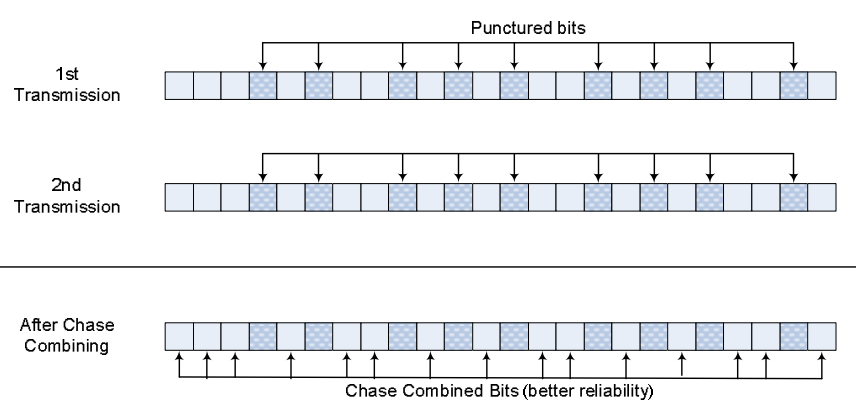

Fig. 1 Chase combining method

\section{B. Incremental Redundancy (IR) combining}

In the IR combining method as shown in Fig. 2, a transmitter that receives a NACK message retransmits the packet which has different punctured data and parity pattern from the original packet. If the receiver receives a retransmitted packet, it combines the original transmitted packet with CRC error with the retransmitted one with different data and parity pattern. At every retransmission, the redundant information bit is increased. The IR combining method improves the receiver performance by increasing redundancy bits for each retransmission.

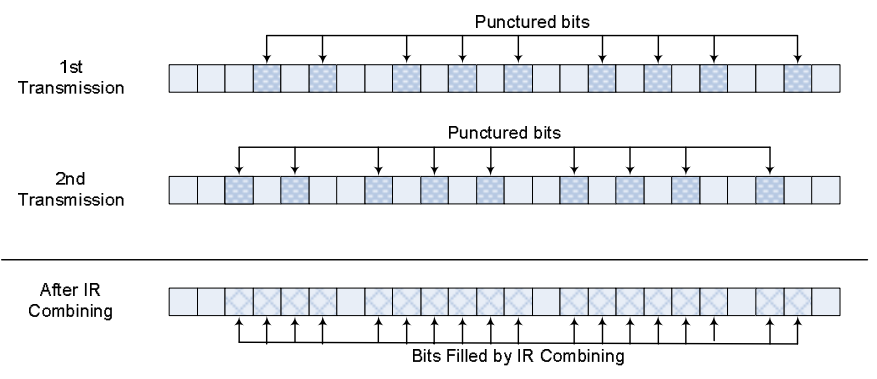

Fig. 2 IR combining method

\section{THE PROPOSED HARQ SCHEME}

In the normal HARQ scheme specified, when the receiver receives a packet it checks whether the received packet is retransmitted or not. If the received packet is turned out not to be a retransmitted one, then it performs the turbo decoding and CRC check processes as shown in Fig. 3. If the CRC check fails, the receiver requests retransmission by sending the NACK message to the BS. If the CRC check is OK, the receiver sends the decoded packet to the reordering buffer for the next stage processing. On the other hand, when the received packet is turned out to be a retransmitted one, the receiver combines the original received packet with error with the retransmitted received packet. After that, it performs the same decoding and following processes as shown in Fig. 3. In this case, if we assume that the retransmitted packet has no error, the simple combining scheme in which the retransmitted packet without error is combined with the original packet with error might results in the poor receiver performance.

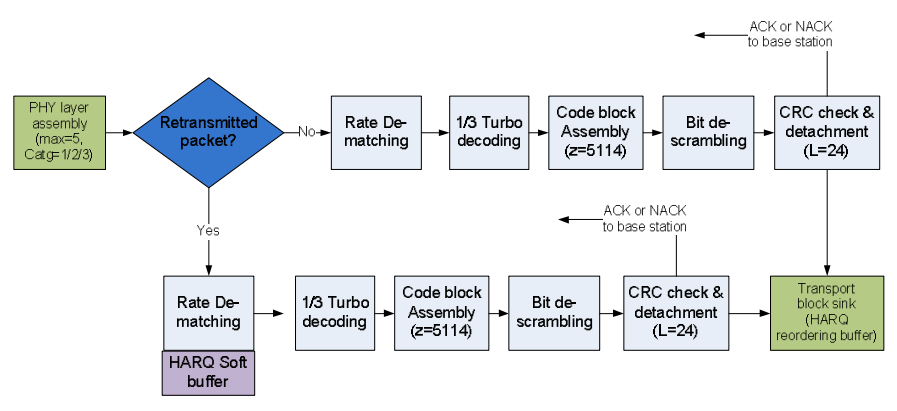

Fig. 3. The normal HARQ scheme using the direct soft combining at a mobile receiver

The decoding process and the CRC check might be performed for a retransmitted packet before combining as shown in Fig. 4. If the CRC check is OK, the receiver sends the decoded packet to the reordering buffer for the next stage processing. If the CRC check fails, the receiver combines the original received packet with error with the retransmitted received packet. Then, the decoding and the CRC check processes are performed [3]. Even though this scheme shows better receiver performance than that in Fig. 3, the computational complexity increases considerably because the decoding process is performed twice if the retransmitted packet is turned out to have errors by failing the CRC check.

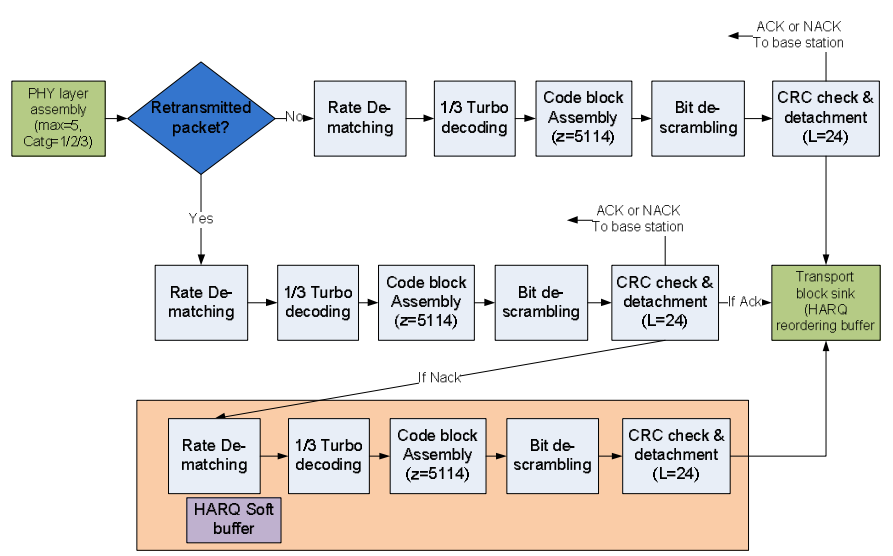

Fig. 4. The HARQ scheme performing the CRC check first and the soft combining later at a mobile receiver 
In order to overcome the high computational complexity of the scheme mentioned above, we propose to exploit the channel condition identified by the CQI number to determine whether only the retransmitted packet is decoded without soft combining or it is combined with the original packet and decoded. The CQI number is related to the ratio of the signal power to the interference and noise power (SINR) as in the HSDPA system specified in [1], where the CQI number ranges from 0 to 30 . Low CQI number corresponds to poor channel condition with low SINR value and high CQI number indicates good channel condition with high SINR value.

In the proposed scheme as shown in Fig. 5, the receiver checks if the received packet is a retransmitted one or not. If the received packet is a new packet, the normal decoding and the following processes are performed. However, if the received packet is turned out to be a retransmitted one, the receiver again checks the channel condition by comparing the measured SINR value with the predetermined threshold. If the SINR is larger than the threshold, the decoding process is performed without soft combining. If the SINR is smaller than the threshold, the retransmitted received packet is soft combined with the original received packet with errors. After that, the decoding process is performed.

This scheme utilizes the channel condition identified by the CQI number to help the receiver determine whether to decode the retransmitted packet directly without combining or to combine the retransmitted packet with the original packet and decode afterward. Since the channel condition is already measured and mapped to the CQI number by the receiver to report to the $\mathrm{BS}$ for the $\mathrm{AMC}$ function, there is no additional increase of computational complexity for measuring the SINR for this scheme. In addition, when the channel condition is bad, this method avoids the first CRC check which is performed in Fig. 4 by utilizing SINR value and roughly determining whether to decode the retransmitted packet only without soft combining or to combine the retransmitted packet with the original packet. As a result, it shows performance improvement compared to the scheme in Fig. 3. And also even though the proposed scheme shows a little performance degradation compared to that in Fig. 4, its computational complexity decreases a lot compared to the scheme in Fig. 4.

\section{SINR ESTIMATION}

The SINR is used to measure the channel quality, which is normally expressed as below,

$$
\operatorname{SINR}(x)=\frac{E[x]^{2}}{\operatorname{Var}[x]}
$$

where $x$ denotes the received signal $x$. And $E[x]^{2}$ indicates the mean power of the received signal and $\operatorname{Var}[x]$ means the variance of the received signal $x$. In the paper, pilot symbols of the common pilot channel (CPICH) specified in [1] is utilized to measure the SINR.

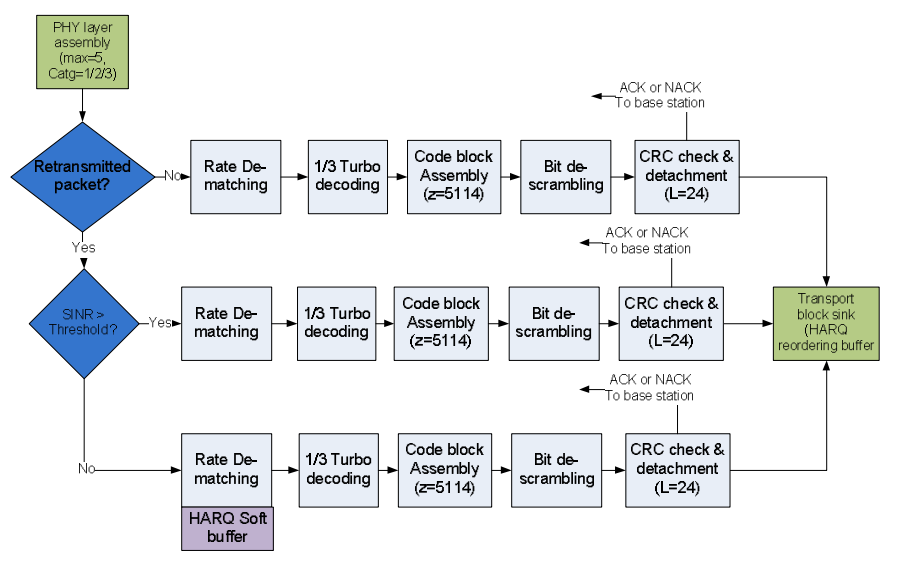

Fig. 5. The selective HARQ scheme choosing the soft combining or direct decoding based on channel conditions

The CPICH is QPSK modulated and the power of pilot symbols in the $\mathrm{CPICH}$ is always constant specified in [1]. Since the mean square of the received pilot symbols is the signal power and the variance of the received pilot symbols corresponds to the interference and noise power, (1) may be expressed in (2) below,

$$
\operatorname{SINR}(x)=\frac{\left|\frac{1}{N} \sum_{k=1}^{N} x_{k}\right|^{2}}{\frac{1}{N} \sum_{k=1}^{N}\left|x_{k}-\frac{1}{N} \sum_{k=1}^{N} x_{k}\right|^{2}}
$$

where $x_{k}$ denotes the discrete value of pilot symbols and $\mathrm{N}$ indicates the number of pilot symbols in a subframe of $5 \mathrm{~ms}$ length.

\section{SIMULATION RESULTS AND ANALYSIS}

We use computer simulations to compare the performance of the proposed scheme with those of the other schemes. The channel models and various parameters of the HSDPA system specified in [4] are used in simulations. Channel models of VA30 and VA120 [4], [6] are used. Modulation type for data signal is 16QAM and the number of multicodes is 4 as specified in [4]. And the IR combining method is used for the HARQ scheme. In the HARQ scheme for the simulation, three retransmissions are allowed. If the NACK occurs after the third retransmission, the BS discards the selected packet and transmits a new packet. The threshold value of 220 for the SINR is used to determine whether to decode the retransmitted packet only without soft combining or to combine the retransmitted one with the original packet and decode afterward as shown in Fig. 5. The threshold above is chosen to satisfy the condition that the probability of the ACK occurrence is almost eighty or ninety percent if the SINR value is larger than the threshold. The ratio of the high speed downlink data channel power over the total transmitter power is expressed as Ec/Ior. And $-3 \mathrm{~dB}$ and $-6 \mathrm{~dB}$ of Ec/Ior 
are used for simulations. Finally H-set 1, H-set 2, and H-set 3 are chosen for simulations.

Fig. 6 to Fig. 8 show the simulation results of the proposed HARQ scheme and other HARQ schemes in terms of data throughput. When Ec/Ior is $-6 \mathrm{~dB}$, the HARQ scheme performing the CRC check first and the soft combining later as shown in Fig. 4 shows the highest throughput (marked with (B)) among three HARQ schemes as expected for both channel models of VA30 and VA120. And the proposed selective HARQ scheme which chooses the soft combining or the direct decoding based on channel conditions as shown in Fig. 5 shows about two to three times better performance(marked with (C)) than that (marked with (A)) of the normal HARQ scheme using direct soft combining as shown in Fig. 3. For the proposed scheme takes the quality of retransmitted packets into account based on channel conditions before performing the soft combining with the original packet with error. However, when Ec/Ior is $-3 \mathrm{~dB}$, which means the power level of the data signal is high enough to cause only a little error which may be easily corrected by the decoding process, any of three HARQ schemes shows almost the same throughput for both channel models of VA30 and VA120 as expected.

\section{CONCLUSION}

We presented a selective HARQ scheme operating based on channel conditions for high speed packet data transmission systems. It first checks the quality of retransmitted packets using SINR value which is related to the channel condition identified by the CQI number before combining the retransmitted packet with the original packet with error. So if the quality of the retransmitted packet is good, this scheme assumes that there is no error in the retransmitted packet and uses only the retransmitted packet for the following signal processing without combining it and the original packet with error. As a result, the proposed selective HARQ scheme shows about two to three times performance improvement in terms of throughput.

In addition, since the channel condition is already measured and mapped to the CQI number by the receiver to report to the $\mathrm{BS}$ for the AMC function, additional increase of computational complexity at the receiver is not necessary for this scheme.

\section{ACKNOWLEDGEMENT}

This research was supported in part by EoNex Technologies, and in part by MIC (Ministry of Information and Communication) \& IITA (Institute for Information Technology Advancement), Korea, through TI-KAIST international joint program conducted by MMPC (Mobile Media Platform Center) of KAIST.

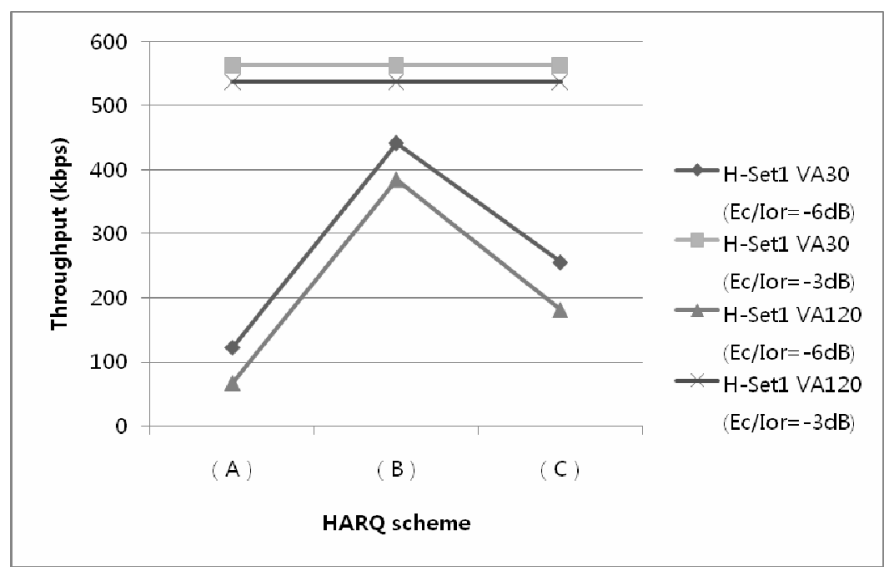

Fig. 6. Throughput performance among three HARQ schemes at $\mathrm{H}$-set 1

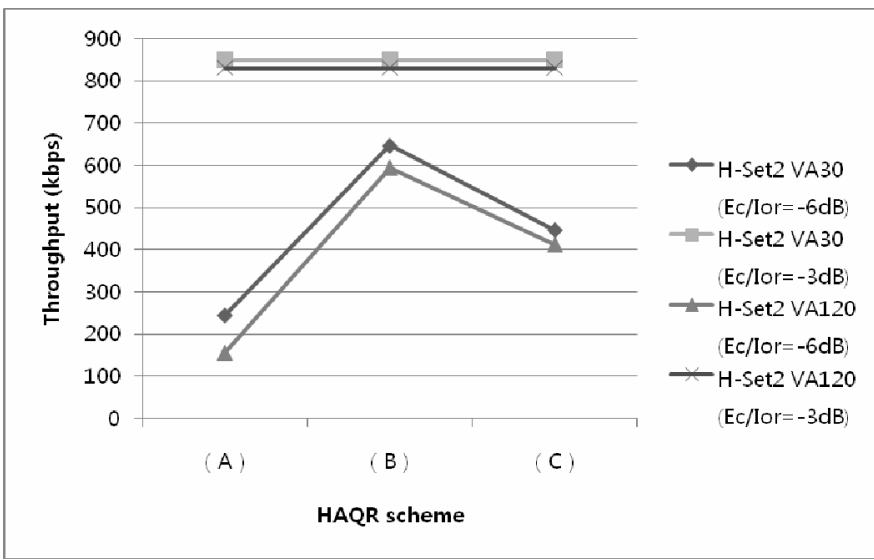

Fig. 7. Throughput performance among three HARQ schemes at $\mathrm{H}$-set 2

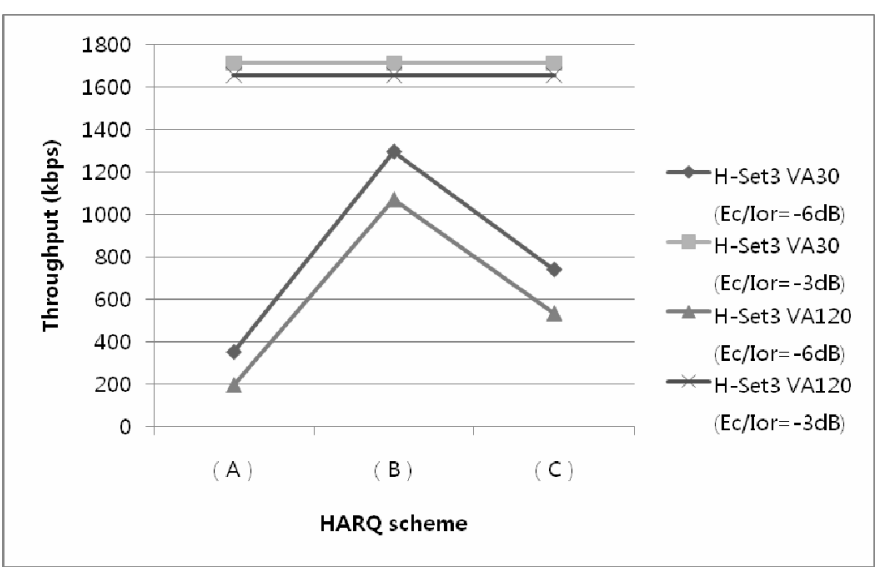

Fig. 8. Throughput performance among three HARQ schemes at $\mathrm{H}$-set 3

\section{REFERENCES}

[1] 3GPP. TS 34.121, V6.1.0, "Radio transmission and reception (FDD)", June 2005.

[2] 3GPP. TS 25.212, V6.5.0, "Multiplexing and Channel Coding (FDD)", June 2005. 
The 18th Annual IEEE International Symposium on Personal, Indoor and Mobile Radio Communications (PIMRC'07)

[3] Minjae Park, Daewon Lee, Moohong Lee and Hwang Soo Lee, "A Concatenated Combining Method for HARQ in the HSDPA System", The 16th Joint Conference on Communications \& Information, Korea, April 2006

[4] 3GPP. TS 25.101, V7.1.0, "User Equipment (UE) radio transmission and reception (FDD)", Sept. 2005.

[5] Frenger, P., Parkvall, S., Dahlman, E, "Performance comparison of HARQ with Chase combining and incremental redundancy for HSDPA", VTC 2001 Fall. IEEE VTS 54th, vol.3, pp. 1829 - 1833.

[6] L.M. Correia. Wireless Flexible Personlised Communications COST 259: European Co-operation in Mobile Radio Research. John Wiley \& Sons Ltd. England. June 2001. 